\title{
The role of surgeons during the COVID-19 pandemic: impact on training and lessons learned from a surgical resident's perspective
}

\author{
Heitham Wady ${ }^{1}$ (1) $\cdot$ David Restle ${ }^{1} \cdot$ Juyeon Park $^{1} \cdot$ Aurora $^{\text {Pryor }}{ }^{2} \cdot$ Mark Talamini $^{1} \cdot$ Sherif Abdel-Misih $^{3}$
}

Received: 13 May 2020 / Accepted: 4 July 2020 / Published online: 14 July 2020

(C) Springer Science+Business Media, LLC, part of Springer Nature 2020

\begin{abstract}
Background Surgeons are trained as "internists that also operate," bringing an important skillset to patient management during the current COVID-19 pandemic. A review was performed to illustrate the response of surgical staff during the pandemic with regard to patient care and residency training.

Methods The evaluation and assessment of the changes enacted at Stony Brook Medicine's Department of Surgery is illustrated through the unique perspective of surgical residents. No IRB approval or written consent was obtained nor it was necessary for the purposes of this paper.

Results Hospital policy was enacted to hinder transmission of COVID-19 and included limited gatherings of people, restricted travel, quarantined symptomatic staff, and careful surveillance for disease incidence. Surgical residency transformed as residents were diverted from traditional surgical services to staff new COVID-19 ICUs. Education transitioned to an online-based platform for lectures and reviews. New skills sets were acquired such as PICC line placement and complex ventilator management.

Conclusions The viral surge impacted surgical training while also providing unique lessons regarding preparedness and strategic planning for future pandemic and disaster management.
\end{abstract}

Keywords COVID $19 \cdot$ Pandemic $\cdot$ Surgeons role $\cdot$ Health policy

During general surgery residency in the United States, residents are expected to learn the foundational sciences of surgical disease, technical surgical skills, perioperative patient care management, and the expected disease courses that afflict surgical patients. Success of operative management comes with the understanding and anticipation of problems with optimal preparedness to handle these challenges. The

Heitham Wady

heitham.wady@stonybrookmedicine.edu

1 Department of Surgery, Stony Brook University Medical Center, Stony Brook, NY, USA

2 Division of Bariatric, Foregut and Advanced Gastrointestinal Surgery, Department of Surgery, Stony Brook University Medical Center, Stony Brook, NY, USA

3 Division of Surgical Oncology, Department of Surgery, Stony Brook University Medical Center, Stony Brook, NY, USA resilience, grit, and ability of surgeons to react to and manage challenges defines the profession.

In March 2020, the Corona Virus Disease 2019 (COVID19) pandemic presented an unprecedented challenge to the infrastructure of surgical care throughout the nation. While health systems have had to dynamically adapt to these changes, surgeons have also worked to utilize their skillsets to aid in crisis, with a transition to roles outside the operating room (OR) and into the intensive care unit (ICU). Within this report, we will describe the effect of the COVID-19 pandemic on the Stony Brook University Hospital's Department of Surgery, the actions surgeons have undertaken in response to this crisis, as well as describe the unique effects on surgical training.

\section{Background}

COVID-19 is caused by SARS-COV2, a non-segmented positive sense RNA virus within the Coronaviridae family $[1,2]$. COVID-19 was first identified in Wuhan, China, as 
a cluster of patients with pneumonia of unknown cause in December of 2019. The first case of COVID-19 diagnosed in the US occurred on January 19, 2020 in Washington [3]. By June 15, 2020, over 7.69 million cases and 427,630 deaths were reported globally, with over $2,063,812$ cases and 115,271 deaths in the United States $[4,5]$.

Clinical manifestations include fever, cough, and dyspnea, around $14 \%$ of patients require hospitalization, and roughly $12 \%$ of patients require an ICU admission [6-8]. Dyspnea develops around 5-8 days from illness onset and mechanical ventilation is typically initiated in severe cases after roughly 10 days from disease onset $[1,9,10]$. While exact routes of transmission have not been identified, other viruses within the coronavirus family are transmitted via respiratory droplets and contact with infected secretions. Reports from China and Singapore cite pre-symptomatic patient transmission rates of $12.6 \%$ and $6.4 \%$, with Dr. Robert Redfield, director of the Center for Disease Control, stating in a recent interview that he estimates up to $25 \%$ of infected persons are asymptomatic [11, 12]. The rapid spread of COVID-19 has crippled healthcare systems globally, and in response strategies have been deployed both institutionally and at society levels to help combat this disease process.

\section{Public health strategies}

"Staying ahead of the Curve" is an epidemiological expression for evaluating a healthcare system's resource capabilities as it relates to disease incidence in the community. As illustrated in Fig. 1, to continue providing optimal patient care during times of crisis requires the concept of "flattening" the curve such that measures are implemented to reduce and delay the peak outbreak of cases [13, 14]. Preventing hospital systems from being overwhelmed by cases can curb mortality rates as resources such as ICU beds and ventilators are finite and exhaustible. A report from the Johns Hopkins Center for Health Security calculated that approximately
160,000 ventilators are available for use in the US [15]. However, the US Department of Health and Human Services in 2005 published a strategic plan in the event of an influenza pandemic similar to that of the Great Influenza of 1918 with ventilator requirement estimates exceeding 700,000 [15-18].

Several public health measures can aide in this endeavor. Social distancing (home schooling, working from home, and self - quarantine) and personal hygiene behaviors (frequent hand washing, cough etiquette, and usage of personal protective equipment (PPE) in high risk environments) can help decrease exposure and disease transmission rates. Identification of exposure to affected individuals, temperature monitoring, and awareness of upper respiratory tract infection (URI) symptoms can aid in early diagnosis, isolation, and treatment. Limiting travel to high risk regions can also decrease exposure risk $[8,10,13]$.

\section{Hospital infection control}

Stony Brook University Hospital (SBUH), located in Suffolk County, Long Island, NY, has been particularly heavily hit with COVID-19 patients. Our county has ranked as high as sixth in the country [19]. As COVID-19 underwent community spread within the US with 62 confirmed cases reported, a hospital incident command system (HICS) was established at SBUH on February 28th [20]. All patients arriving via the Emergency Room, Ambulatory Care Centers, and Outpatient Clinics were screened with questions focused on symptom identification, travel history, and exposure risks. Patients with URI symptoms were directed to wear standard surgical masks and Persons Under Investigation (PUI) were placed on respiratory isolation precautions with notification made to the Department of Health (DOH). By March 10th the total cumulative cases in the US rose to 696 and the Visitor Policy, guided by Department of Health
Fig. 1 "Flattening the Curve" Depiction illustrating how slowing disease transmission can help prevent hospital overcapacity (Adapted from: CDC. Interim pre-pandemic planning guidance: community strategy for pandemic influenza mitigation in the United States-early, targeted, layered use of nonpharmaceutical interventions. Atlanta, GA: US Department of Health and Human Services, CDC 2007. https://stacks.cdc. gov/view/cdc/11425)

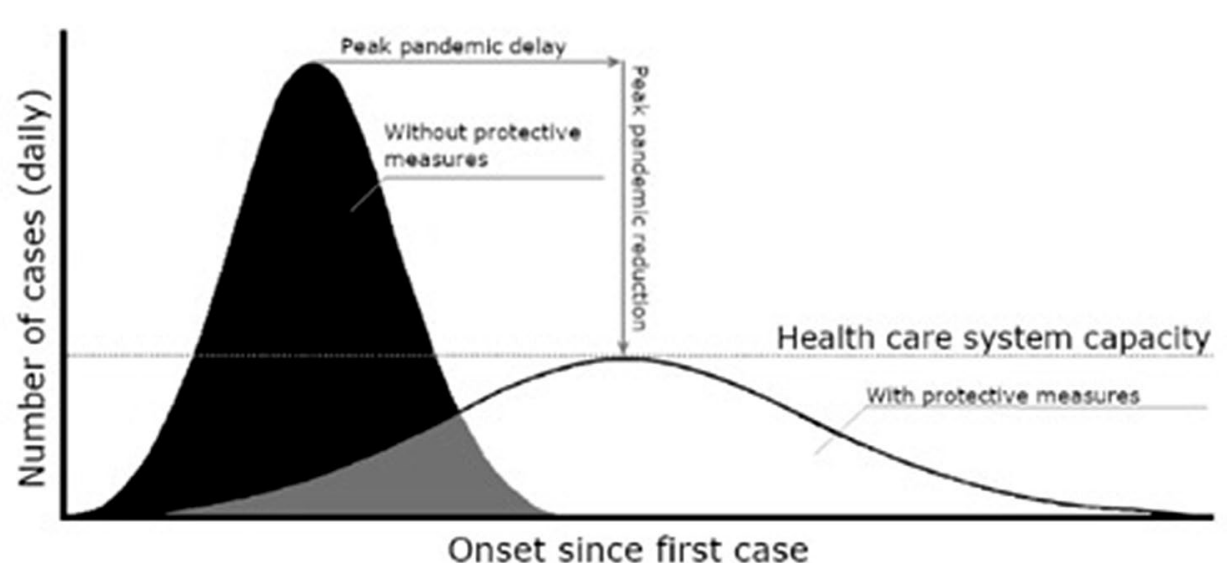


guidelines, was modified to minimize the amount of traffic in the hospital [20].

Areas in the hospital were identified for creation of additional beds (both ICU and medical) and additional ventilators were requisitioned. The Ambulatory Care Pavilion (outpatient clinical area) was transformed into a Triage Area to assess low acuity patients with URI symptoms. New York State partnered with SBUH to initiate on campus drive-through testing. Social distancing was employed and number limits for personnel within communal areas was strictly enforced. Electronic communication platforms were initiated to provide hourly updates on management plans and minimize person to person contact. Outpatient clinics were transitioned to telehealth as possible, with physical distancing and PPE measures employed for in-person appointments. A summary of hospital infection control measures is listed in Table 1.

\section{Travel, isolation, quarantine}

To protect the hospital workforce and limit loss of providers due to infection, a hospital wide mandate was implemented banning residents from professional travel for conferences. Symptomatic residents were tested and self-quarantined until results could verify disease status. Those testing positive with mild symptoms were instructed to maintain self-quarantine. To return to work, a resident must maintain isolation for at least 7 days from illness onset, be fever free for $72 \mathrm{~h}$ without antipyretic use, show symptom improvement, and wear a facemask while working for 14 days from symptom onset. Figure 2 provides a flow chart algorithm used by healthcare personnel who became symptomatic.

\section{Surgery policies affecting $O R$, resident scheduling}

On March 13th the total cumulative cases in the US doubled to 1,264 and the American College of Surgeons recommended canceling or postponing all elective surgeries to decrease the flow of patients into hospitals [20, 21]. On March 17, the surgical departments at SBUH suspended all elective cases. Urgent or semi-urgent cases continued to be performed until March 23rd as the total cases of COVID-19 in the US soared to 31,573 . With the rapidly increasing number of PUIs and confirmed COVID-19 infected patients, the need for resident coverage in normally non-surgical fields was ever growing.

As a result, the Surgery department re-organized their schedule to provide coverage for surgical and non-surgical services, adhering to new requirements given the pandemic. Three resident teams were created, a daytime and nighttime call team and a backup team ready to rotate in as residents became ill. This system of multiple surgical call teams, including a backup team, has been described at other institutions [22]. As the hospital approached surge capacity, more surgical residents were enlisted to serve on non-surgical rotations and within days, $>90 \%$ of all resident staff were volunteered to aid in patient care on COVID-19 Units.

\section{Surgeon's role in COVID-19 pandemic}

"There are no more surgeons, urologists, orthopedists, we are only doctors who suddenly become part of a single team to face this tsunami that has overwhelmed us" [23]. Dr. Daniele Macchini, in a social media post that was widely distributed described the atmosphere during the pandemic in Italy. He illustrated the necessity to have surgeons fill the void created by a wave of high acuity patients and a shortage of intensivists available to treat them.

To this end, the response of the Department of Surgery at Stony Brook has been profound. Surgical staff have volunteered their efforts to staff COVID-19 Units to treat these complex patients. Surgical clinics were almost entirely transformed to telehealth care visits to allow for continued outpatient care. Surgeries were limited to emergent cases only, allowing for redeployment of anesthesia staff to inpatient hospital floors. This reduced the inpatient surgical service census, allowing for additional ventilator support availability

Table 1 Summarized hospital infection control measures

Hospital infection control measures

Establishment of Hospital Incident Command System

Patients arriving at Stony Brook Facilities screened for possible exposure

Persons Under Investigation placed on Respiratory Precautions

Creation of additional beds and additional ventilators requisitioned

Social Distancing and Number Limits in Communal Areas strictly enforced

\author{
Visitor Policy restricted to limit traffic into the hospital \\ Ambulatory Care Pavilion transitioned to Triage Center for suspected \\ patients \\ Outpatient Clinics transitioned to telehealth
}


Fig. 2 Algorithm for addressing healthcare personnel who became symptomatic during COVID-19 Pandemic

\section{Hospital Personnel}


Self-quarantined and tested for COVID-19

\section{Maintain self-quarantine for 7 days from symptom onset, fever free for $72 \mathrm{hrs}$, show symptom improvement, and wear facemask for 14 days from symptom onset}

for the COVID-19 patient census. OR and PACU personnel aided in staffing new COVID-19 patient units. Our department advocated for institutional scrubs that could be used inclusively by all hospital medical staff, thus reducing the risk of virus transmission outside the hospital. Spurred by family and friends of residents, outreach efforts made to the local community led to unanticipated generous donations of N95 masks, surgical face masks, and food to aid the SBUH pandemic efforts.

During times of crisis, communication is of paramount importance to ensuring an effective response. The Surgery Department utilized information technology to conform to requisite physical distancing while maintaining social communication. Daily patient hand offs and information meetings were continued using secure video conferencing and messaging applications. Our division heads met regularly, email updates on hospital policy were sent regularly, and our Chair delivered daily morning voice message updates maintaining institutional transparency and keeping us informed of the efforts to date and anticipated future obstacles. Our Program Director served as a bridge between the administrative chief residents and hospital administration, and administrative chief residents served as liaisons for the residents. A summary of various roles of the surgeon during the pandemic is provided in Table 2.

Table 2 The surgeon's role in the COVID-19 pandemic

Surgeon's role in COVID-19 pandemic

Surgical clinics transformed to telehealth care visits

Anesthesia staff redeployed to inpatient hospital floors

Institutional scrubs used inclusively by all hospital staff to decrease viral transmission

Information technology used for patient hand offs, daily meetings, and for education platforms
Surgeries limited to emergent cases only

Reduced surgical inpatient census allowed for additional ventilator support availability

Outreach efforts lead to donations of N95 masks, surgical face masks, and food to aid in efforts

Clear communication from senior staff and administrators 


\section{Challenges faced through unique crisis}

The turmoil created by COVID-19 presented many clinical, systems, and educational challenges. Among them was need for rapid turnaround on COVID-19 test results. As the pandemic infiltrated our healthcare system, PUIs had their tests sent to an outside lab with a turnaround time of $>5$ days. Healthcare providers with symptoms were required to stay home until testing returned negative. Delays in test results equated to fewer personnel available for patient management. Ultimately, our pathology department was able to set up in house testing for rapid results.

The hardships of the pandemic are not solely the burden of healthcare workers. For the family of a critically ill patient with COVID-19 left in isolation, the process is particularly difficult. The family needs frequent communication from care teams. Assigning hospital staff to speak with family members in the absence of visitation ability may be required to offset this. At our institution, care teams regularly update patient families on a daily basis with changes in a patient's clinical status.

\section{Lessons learned}

The need for N95 respirators, PPE, and ventilators has been well documented through the COVID-19 crises, but other challenges were encountered through the SBUH COVID-19 pandemic management. Essential resources including physical space and bed availability can be overlooked when facing capacity and disease surge issues. While SBUH was effective in expanding hospital units into floors for critically ill COVID-19 patients, other critical resources were discovered to be essential. These include ultrasound technology to aid in central and arterial lines for access, as well as certain cardiac and respiratory medications. Medications crucial to the care of the COVID-19 patient population include sedatives (ex: propofol, fentanyl, and dexmedetomidine) and vasopressors (ex: vasopressin and norepinephrine). Adequate supply chain of these hospital resources are paramount and should be readily available as delays in obtaining them can lead to delays in patient care.

The treatment of ICU patients requires constant diligence and monitoring of respiratory and hemodynamic parameters. Essential information is obtained by viewing a patient's ventilator settings and bedside vitals. In the setting of a viral pandemic, conserving PPE equipment and reducing viral exposure is accomplished by limiting the number of visits into a patient's room. Thus, the vitals monitor and respirator should be facing the door to allow monitoring of both respiratory and hemodynamic parameters from the outside. When plausible, lengthened peripheral intravenous lines should be considered to allow IV poles to be located outside patient rooms making them readily accessible to ICU staff without need for PPE and close contact transmission risks.

Guided by our surgical intensivist team, clinical but anecdotal experience noted that after approximately 7-10 days, some intubated patients experienced increased peak airway pressures, peak inspiratory pressures, and episodes of hypoxemia with difficulties in ventilation. It was postulated that the endotracheal tubes (ETT) were occluding secondary to bronchial tracheal sloughing and debris that was unable to be suctioned out [24]. As such, a surgical intensivist driven ETT exchange protocol was developed with a dedicated ETT exchange team created to treat patients with and prevent clogged ETTs. In the majority of cases where ETT exchange was performed, improvements in oxygenation and ventilation were observed.

As central venous access became essential in many cases for ICU supportive care, a Peripherally Inserted Central Catheter (PICC) service was created by the vascular surgery division. In addition to traditional surgical resident access skills, this required a protocol driven training and credentialing of surgical residents to place PICC lines. This training provided surgical residents with a skillset not ordinarily required or obtained in general surgery training. Based on patient stability and intubation status, PICC lines were placed by a dedicated credentialed staff of residents and fellows who completed an online didactic course followed by supervised training of PICC placement by already credentialed personnel. This PICC team allowed for rapid placement of PICCs to circumvent central venous catheter placement, with the added benefit of having central access for longer durations.

During the early course of our COVID-19 experience, infected patients appeared to have pro-inflammatory and prothrombotic responses due to the infection, with elevations in inflammatory markers and abnormal coagulation parameters $[25,26]$. As the role of anticoagulation in relation to coagulopathy from infection evolved, anticoagulation treatment algorithms were pioneered by our vascular surgery division in collaboration with our surgical intensivists. These protocols were adopted institution wide to help guide therapy in the hopes of diminishing end organ damage and mortality [27]. Follow-up of the prothrombotic effects of COVID-19 infection and the effects of the anticoagulation protocols are being investigated as we continue to learn and better understand the pathophysiology. A summary of the lessons learned while managing COVID-19 patients is provided in Table 3. 
Table 3 Lessons Learned while dealing with COVID-19 Pandemic

\begin{tabular}{ll}
\hline Encountered problem & Lesson learned \\
\hline Overlooked critical resources needed for COVID units & Vasopressors, Sedatives, Ultrasound technology should be readily available \\
Limited PPE equipment and constant use for entering patient rooms & Vitals monitor and respirator should be facing door, lengthened peripheral \\
& IV lines so IV poles can be outside of room \\
Occluded ETTs and difficulty with ventilation & Creation of teams for early ETT exchange \\
Need for long term central IV access & Training and credentialing of surgical residents for PICC placement \\
Prothrombotic effects of infection & Adoption of anticoagulation protocol \\
\hline
\end{tabular}

\section{Discussion}

In the setting of a unique health crisis posed by a potent adversary like COVID-19, the SBUH leadership and Hospital Incident Command System were critical to the institution and standardization of care protocols and systems-related patient care management (beds, resources, ventilators, PPE) strategies. This was especially important given the clinical volume faced with SBUH being the major academic tertiary care center providing care to Suffolk County. The Johns Hopkins Coronavirus Resource Center stated that Suffolk County had up to the 6th most confirmed cases by county [19]. During the SBUH response, surgery personnel took advantage of their expertise to provide critical insights into the care of these patients. Specifically, the development of a line access service, anticoagulation protocol, and an endotracheal tube exchange protocol in addition to the excellent supportive care have been critical to the positive outcomes seen in the SBUH COVID-19 patients.

Senior surgical residents are provided excellent education, exposure, and experience in ICU training during their 2 nd and 3rd years of residency while rotating in the Surgical ICU and Cardiothoracic ICU. During these months, residents are taught extensively about ventilator care, respiratory mechanics, vasopressors, fluid and electrolyte management, cardiac arrhythmias, and types of sedatives. In preparation of the COVID-19 Pandemic, senior surgical intensivists provided additional refresher courses on critical care management for both residents and faculty. While residents were given the autonomy to treat and manage these complex patients, close guidance by surgical intensivists provided a safe barrier for both education and supervision. Procedures such as central and arterial line placement were performed by credentialed residents who learned about proper procedural technique, necessary equipment, and ultrasound technology from senior staff.

The response met by the Department of Surgery at Stony Brook has provided a template that can be used by other hospitals to enable surgeons to combine their technical and clinical skillsets in a collaborative effort to manage patients afflicted by COVID-19. Our keys to success have been simple: Leadership has been transparent and informative, providing real time data and tools to enable surgeons to transition smoothly from surgical to medical floors. Residents have been resilient and unwavering in their commitment to patient care, and fearless in their battle with this disease. The hospital staff and community have come together in supportive roles, understanding that through unity and perseverance we will turn darkness into light.

Throughout this process, the constant barrage of fear for the ill and uncertainty of the future created a hyperacute environment of stress. The surgical residents took measures to offset these negative forces. We organized social events in the form of virtual happy hours, using electronic platforms to stay connected in a friendlier environment, purchased snacks for our colleagues who were on overnight shifts, and grocery shopped for each other due to the demands of the schedule.

On a personal note, this pandemic has reaffirmed our resident authors' dedication to the field of surgery. Through our technical skills and critical care training we have been able to help care for so many critically ill patients. The culmination of all that encompasses surgical residency training has allowed us to move forward and face COVID-19 head on. While traditional surgical training was disrupted, we had the opportunity to gain invaluable knowledge in ventilator management, infectious disease protocols, and disease transmission vectors. This novel experience exemplified the continuing art of medicine where the foundational sciences background and astute clinical observations positively impacted patient outcomes despite the lack of statistical significance to guide treatment. Where others may have wavered, we marched on in a collaborative, multidisciplinary fashion to confront this pandemic. To our fellow surgical residents facing the daunting test of this pandemic, I encourage you to move forward with the comfort in knowing your training has prepared you to face the challenges of tomorrow, whether it be in the operating room or ICU.

Acknowledgements We acknowledge the consultation and support of the Department of Surgery faculty and residents as well as Stony Brook University Hospital Senior Administration officials.

Funding No sources of funding obtained for this manuscript. 


\section{Compliance with ethical standards}

Disclosures Drs. Heitham Wady, David Restle, Juyeon Park, Sherif Abdel-Misih, and Mark Talamini have no conflicts of interest or financial ties to disclose. Dr. Aurora Pryor receives honoraria for speaking from Gore, Merck, and Stryker, receives research support from Obalon, and is a consultant for Medical Devices Business Services.

\section{References}

1. Huang C, Wang Y, Li X, Ren L, Zhao J, Hu Y et al (2020) Clinical features of patients infected with 2019 novel coronavirus in Wuhan, China. Lancet 395(10223):497-506. https://doi. org/10.1016/S0140-6736(20)30183-5

2. Lai C, Shih T, Ko W, Tang H, Hsueh P (2020) Severe acute respiratory syndrome coronavirus 2 (SARS-CoV-2) and coronavirus disease-2019 (COVID-19): the epidemic and the challenges. Int J Antimicrob Agents 55(3):105924. https://doi.org/10.1016/j.ijant imicag.2020.105924

3. Holshue M, DeBolt C, Lindquist S (2020) First case of 2019 novel coronavirus in the United States. NEJM. https://doi.org/10.1056/ NEJMoa2001191

4. Coronavirus Disease 2019 (COVID-19) situation report -99. World Health Organization. WHO. https://www.who.int/docs/ default-source/coronaviruse/situation-reports/20200428-sitre p-99-covid-19.pdf?sfvrsn=119fc381_2. Accessed 28 Apr 2020

5. Cases in U.S. Centers for Disease Control and Prevention. CDC. https://www.cdc.gov/coronavirus/2019-ncov/cases-updates/cases -in-us.html. Accessed 12 Apr 2020

6. Maclaren G, Fisher D, Brodie D (2020) Preparing for the most critically Ill patients with COVID 19: the potential role of extracorpeal membrane oxygenation. JAMA 323(13):1245-1246. https ://doi.org/10.1001/jama.2020.2342

7. Phua J, Weng L, Ling L, Egi M, Lim C, Divatia JV, Shrestha BR et al (2020) Intensive care management of coronavirus disease 2019 (COVID-19): challenges and recommendations. Lancet Respir Med. https://doi.org/10.1016/s2213-2600(20)30161-2

8. Team NCPERE (2020) Vital surveillances: the epidemiological characteristics of an outbreak of 2019 novel coronavirus diseases (COVID-19) - China. China CDC Wkly 2(8):113-122

9. Zhou F, Yu T, Du R, Fan G, Liu Y, Liu Z, Xiang J et al (2020) Clinical course and risk factors for mortality of adult inpatients with COVID-19 in Wuhan, China: a retrospective cohort study. Lancet 395(10229):1054-1062. https://doi.org/10.1016/s0140 $-6736(20) 30566-3$

10. Li R, Pei S, Chen B, Song Y, Zhang T, Yang W, Shaman J (2020) Substantial undocumented infection facilitates the rapid dissemination of novel coronavirus (SARS-CoV2). Science. https://doi. org/10.1126/science.abb3221

11. Du Z, Xu X, Wu Y, Wang L, Cowling BJ, Meyers LA (2020) The serial interval of COVID-19 from publicly reported confirmed cases. Emerg Infect Dis. https://doi.org/10.3201/eid2606.200357

12. Wei WE, Li Z, Chiew CJ, Yong SE, Toh MP, Lee VJ (2020) Presymptomatic transmission of SARS-CoV-2 - Singapore, January 23-March 16, 2020". MMWR Morb Mortal Wkly Rep 69(14):411-415. https://doi.org/10.15585/mmwr.mm6914e1

13. CDC (2007) Interim pre-pandemic planning guidance: community strategy for pandemic influenza mitigation in the United Statesearly, targeted, layered use of nonpharmaceutical interventions. Atlanta, GA: US Department of Health and Human Services, CDC; 2007. https://stacks.cdc.gov/view/cdc/11425. Accessed 12 Apr 2020

14. Fineberg HV, van Doremalen N, Cao B, Holshue ML, Institute of Medicine (2015) Pandemic preparedness and response - lessons from the H1N1 influenza of 2009: NEJM. N Engl J Med 370:1335
15. Johns Hopkins Center for Health Security. Ventilator stockpiling and availability in the US. Johns Hopkins Bloomberg School of Public Health. https://www.centerforhealthsecurity.org/resources/ COVID-19/200214-VentilatorAvailability-factsheet.pdf. Accessed $14 \mathrm{Feb} 2020$

16. US Department of Health and Human Services. Supplement 7: antiviral drug distribution and use. In: HHS Pandemic Influenza Plan. Washington, DC: HHS; 2005. https://www.cdc.gov/flu/-pdf/profe ssionals/hhspandemicinfluenzaplan.pdf. Accessed 24 Mar 2020

17. Rubinson L, Vaughn F, Nelson S, Giordano S, Kallstrom T, Buckley T, Burney T, et al (2013) Mechanical ventilators in US Acute Care Hospitals: disaster medicine and public health preparedness. Cambridge Core. Cambridge University Press. https://www.cambridge. org/core/journals/disaster-medicine-and-public-health-preparedne ss-/article/mechanical-ventilators-in-us-acute-care-hospitals/F1FDB ACA53531F2A150D6AD8-E96F144D. Accessed 12 Apr 2020

18. Ajao A, Nystrom SV, Koonin LM, Patel A, Howell DR, Baccam P, Lant T, Malatino E, Chamberlin M, Meltzer MI (2015) Assessing the capacity of the US Health Care System to use additional mechanical ventilators during a large-scale public health emergency. Disaster medicine and public health preparedness. U.S. National Library of Medicine. https://www.ncbi.nlm.nih.gov/pmc/ articles/PMC46-36910/. Accessed 12 Apr 2020

19. COVID-19 United States Cases by County. Johns Hopkins Coronavirus Resource Center. https://coronavirus.jhu.edu/us-map. Published April 28, 2020. Accessed 29 Apr 2020

20. Elflein J (2020) U.S. COVID-19 cases by day. https://www.stati sta.com/statistics/1103185/cumulative-coronavirus-covid19-cases -number-us-by-day/. Accessed 15 June 2020

21. American College of Surgeons (2020) American College of Surgeon's releases recommendations for surgical management of elective operations during COVID-19 pandemic. American College of Surgeons Press Release

22. Williams EJ (2020) Coronavirus pandemic and the U.S. surgeon: a dilemma with many facets. Association of Women Surgeons RSS. https://blog.womensurg-eons.org/awschat/coronaviruspand emic-surg/. Accessed 3 Apr 2020

23. Macchini D (2020) Testimony of a surgeon working in Bergamo, in the Heart of Italy's Coronavirus Outbreak. MR Online. https ://mronline.org/2020/03/10/t-estimony-of-a-surgeon-working-inbergamo-in-the-heart-of-italys-coronavirus-outbreak/. Accessed 3 Apr 2020

24. Rubano JA, Jasinski PT, Rutigliano DN et al (2020) Tracheobronchial slough, a potential pathology in endotracheal tube obstruction in patients with coronavirus disease 2019 (COVID-19) in the intensive care setting. Ann Surg. https://doi.org/10.1097/ SLA.0000000000004031

25. Tang N, Li D, Wang X et al (2020) Abnormal coagulation parameters are associated with poor prognosis in patients with novel coronavirus pneumonia. J Thrombos Haemost 18(4):844-847. https://doi.org/10.1111/jth

26. Bikdeli B, Madhavan MV, Jiminez D et al (2020) COVID-19 and thrombotic or thromboembolic disease: implications for prevention, antithrombotic therapy, and follow-up. J Am Coll Cardiol. https://doi.org/10.1016/j.jacc.2020.04.031

27. Tassiopoulos AK, Rubano JA, Rutigliano D et al (2020) Stony Brook anticoagulation protocol for COVID-19 patients. https:// www.sages.org/wp-content/uploads/2020/04/SB-Anticoagulation -Plan-for-COVID-19-patients.pdf. Accessed 26 Apr 2020

Publisher's Note Springer Nature remains neutral with regard to jurisdictional claims in published maps and institutional affiliations. 